\title{
Organisationssoziologie und Bildungsforschung im Dialog
}

\section{Maja Apelt im Gespräch mit Justin Powell}

\author{
Maja Apelt und Justin J. W. Powell
}

\section{Zusammenfassung}

Justin Powell wurde von Maja Apelt zum Verhältnis der Bildungs- zur Organisationssoziologie interviewt. Konkret geht es um Berührungspunkte von Bildungsforschung und Organisationssoziologie, empirische und theoretische Lücken der Organisationssoziologie aus Sicht der Bildungsforschung und zukünftige gemeinsame Fragestellungen.

\section{Schlüsselwörter}

Organisation $\cdot$ Bildungssoziologie $\cdot$ Bildung $\cdot$ Hochschulsystem $\cdot$

Neoinstitutionalistischer $\cdot$ Interview

\section{Welche Berührungspunkte haben Sie zur Organisationssoziologie und welche sehen Sie zwischen Organisations- und Bildungssoziologie?}

Die Vertreterinnen und Vertreter der Organisationssoziologie waren für mich als amerikanischen neo-institutionalistischerem Bildungssoziologen immer sehr einflussreich, denn ich bin in einem Land mit einem sehr stark ausdifferenzierten und stratifizierten Bildungs- und Hochschulsystem aufgewachsen. Deshalb standen Organisationen immer im Zentrum des Interesses; sie sind vielfältige Objekte für

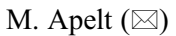

Wirtschafts- und Sozialwissenschaftliche Fakultät, Universität Potsdam, Potsdam, Deutschland E-Mail: maja.apelt@uni-potsdam.de

J. J. W. Powell Institute of Education and Society, University of Luxembourg, Esch-sur-Alzette, Luxembourg E-Mail: justin.powell@uni.lu 
die Forschung. Nachdem ich nach Deutschland kam, habe ich festgestellt, dass es kein so stark ausgeprägtes Interesse der Bildungssoziologie an der Organisationsforschung bzw. an dieser Analyseebene besteht, vielmehr standen entweder Systemoder Individualebene im Fokus. Möglicherweise hängt diese Sichtweise aber auch mit meinem persönlichen Werdegang zusammen, da ich am Max-Planck-Institut für Bildungsforschung mit seinem mikrosoziologischen Zugang, der auf die Bildungsund Beschäftigungskarrieren und Lebensverläufe ausgerichtet ist, promoviert habe. Aufgrund der hierarchischen Gliederung der Schulformen und der Trennung der organisationalen Felder in Deutschland, bspw. der allgemeinen Schulbildung von sonderpädagogischen Fördersystemen (mit dem immer noch dominanten Sonderschulwesen) oder der Berufs- und Hochschulbildung, sind Fragen der Organisationsformen und -felder in meiner Arbeit immer wichtiger geworden: es geht einerseits um das Verhältnis der Felder zueinander und ihren Wechselwirkungen und anderseits um den Status und Wandel der Organisationsformen. Dabei beziehe ich mich auf die zentralen Erkenntnisse der Organisationssoziologie, insbesondere die vielfältigen Arbeiten der Stanford School von John Meyer und Richard Scott sowie auf klassische Beiträge unter anderem von Paul DiMaggio und Walter Powell (DiMaggio und Powell 1983, 1991; Meyer und Rowan 2002; Powell und DiMaggio 1991; Scott 1995). Der einflussreiche World Polity-Ansatz (Meyer et al. 1977) hat die globale Expansion aller Ebenen des Bildungssystems aufgezeigt und hat insbesondere internationale, komparative und Mehrebenenanalysen vorangetrieben.

Sie haben hier bereits feldtheoretische Konzepte angesprochen. Gibt es außerdem noch andere organisationstheoretische Konzepte, mit denen Sie in der Forschung konfrontiert werden, die Sie für Ihre eigene Forschung nutzen oder die aus Ihrer Perspektive heraus für die Bildungssoziologie bedeutsam sind?

Die Bildungssoziologie - mit ihrem Kerngegenstand von Schulen und Hochschulen - kann nicht nur, sie muss mit den Konzepten der Organisationssoziologie arbeiten, gerade wenn es um die Rahmenbedingungen für organisierte Lehr- und Lernprozesse geht. Als Forscher, der in den Vereinigten Staaten sozialisiert wurde, habe ich stärker als viele Kolleginnen und Kollegen im deutschsprachigen Raum meine Grundlagenforschung zur Institutionalisierung von Bildungs- und Wissenschaftssystemen darauf ausgerichtet, auch pragmatische und anwendungsbezogene Analysen mit Implikationen für die Bildungspolitik und -programmatik zu entwickeln. Ich habe deshalb viel in den Versuch, Entwicklungsprozesse zu verfolgen und daraus Lösungsansätze abzuleiten sowie in Transferpublikationen investiert. Am WZB habe ich beispielsweise nach Vorbild der policy briefs von Brookings oder der OECD die digitale Serie WZBriefe (Holtmann et al. 2018) etabliert, um aktuelle Forschungsergebnisse in kompakter Form der breiten Öffentlichkeit zugänglich zu machen. Und gerade bei Fragen des institutionellen oder organisationalen Wandels wäre es für die Bildungssoziologie wichtig, stärker mit den Konzepten der Organisationssoziologie zu arbeiten. Eine solche Forschung zwingt zur expliziten Analyse 
verschiedener Organisationsmerkmale, etwa dem Gründungszeitpunkt der Organisation, ihrer Größe oder ihrer Vernetzung im Feld. Diese entscheidenden Merkmale sind von Belang für wissenschaftliche Erkenntnisse wie auch bildungspolitische Entscheidungen.

\section{Sehen Sie da auch Korrekturbedarf und offene Felder für die Organisa- tionsforschung?}

Ja, den sehe ich durchaus. Zum Beispiel haben Regula Julia Leemann, Christian Imdorf, Michael Sertl und ich gemeinsam mit Raimund Hasse 2014 eine Tagung „Organizing Education: Sociological Approaches, Analysis and Findings" in Basel organisiert - eine Veranstaltung der drei deutschsprachigen Soziologieverbände, die den Versuch eines Brückenschlag zwischen den Teilgebieten der Bildungs- und Organisationssoziologie unternommen hat. Daraus entstand der Sammelband „Die Organisation von Bildung "(Leemann et al. 2016a), in dem wir Analysen zu Schule, Berufsbildung, Hochschule und Weiterbildung zusammengebracht haben. Insbesondere die Überblicksbeiträge von Moritz Rosenmund und Raimund Hasse geben wichtige Einblicke in den aktuellen Stand der Forschung an der Schnittstelle Bildung und Organisation. Wir konstatieren, dass trotz der Durchdringung der Lebensläufe mit organisierter Bildung und der damit verbundenen Omnipräsenz von Bildungsorganisation im Alltag auf Seiten der Bildungssoziologie Deutschlands, Luxemburgs, Österreichs und der Schweiz, organisationsspezifische Fragen bisher nicht im Zentrum der wissenschaftlichen Aufmerksamkeit standen (Leemann et al. 2016b). Das beschreibt in knapper Form die aktuelle Beziehung zwischen diesen beiden Teilgebieten der deutschsprachigen Soziologie.

Die deutschsprachige Bildungssoziologie, denke ich, müsste viel expliziter auf die organisationsbezogenen Wissenschaften eingehen als bisher. Dass dies nicht getan wird, ist gerade vor dem Hintergrund der Entwicklung in der anglofonen Literatur überraschend, die eine Organisationsformen übergreifende und makrokomparative Forschung betreibt. Es sollte meines Erachtens verstärkt die Mesoebene in bildungssoziologischen Studien einbezogen werden, da sich genau dort das Scharnier zwischen Bildungs- und Organisationssoziologie befindet. So habe ich mit Kolleginnen und Kollegen das sich wandelnde Verhältnis von Berufs- und Hochschulforschung in den Blick genommen, in dem vor allem Fragen der Durchlässigkeit und Ungleichheit brisant sind. Es sind weitere Anstrengungen nötig, um die starke Schulbezogenheit der Teildisziplin zu relativieren und neue Fragen zu bearbeiten. Im Rahmen der Sektion Bildung und Erziehung der DGS finden immer wieder Tagungen statt, die zu Sammelbänden in der Reihe der bildungssoziologischen Beiträge bei Beltz Juventa führen. So zum Beispiel zu Themen wie Hochschulbildung, Berufsbildung, Weiterbildung sowie zur Wissenschaft.

Wenn ich vielleicht eine Perspektive ergänzen darf, worin sich die deutschsprachige Organisationssoziologie weiterentwickeln könnte. Gerade die Vielfalt der bildungsbezogenen Organisationsformen bietet Möglichkeiten des Vergleichs in 
Hinblick auf aktuelle Fragen der Internationalisierung, Ökonomisierung, der Privatisierung und der Governance. Diese Themen werden in der vergleichenden Bildungsforschung inzwischen verstärkt berücksichtigt und würden von einem stärker organisationssoziologischen Blick profitieren. So sind die Inklusive Bildung, die Berufsbildung oder außerschulische Bildungsträger gewinnbringende Forschungsfelder, die noch nicht ausreichend aus einer Organisationsperspektive erforscht worden sind. Nicht zuletzt trifft das auch auf meinen eigenen Bereich der feldübergreifenden und international komparativen Studien zu, die die institutionelle und organisationale Komplexität von komplementären Bildungs- und Wissenschaftssystemen analysieren. In Zeiten der fortschreitenden Globalisierung werden Vergleiche (PISA, Sustainable Development Goals, Hochschulrankings \& Co.) immer wichtiger. Dabei sollten wir die vielfältigen Aufgaben der Komparatistik nicht den Ratingagenturen überlassen, sondern selbst kritisch und fundiert bildungs- und organisationssoziologisch analysieren.

Ich danke Ihnen ganz herzlich für den interessanten Einblick.

\section{Literatur}

DiMaggio, Paul J., und Walter W. Powell. 1983. The iron cage revisited. Institutional isomorphism and collective rationality in organisational fields. American Sociological Review 48(2): 147-160.

DiMaggio, Paul J., und Walter W. Powell, Hrsg. 1991. The new institutionalism in organizational analysis. Chicago: University of Chicago Press.

Holtmann, A. C., L. Menze, und H. Solga. 2018. WZBrief Bildung 36/2018. Unentdeckte Kompetenzen. Jugendliche ohne Mittleren Schulabschluss finden schwer einen Ausbildungsplatz. https://www.wzb.eu/en/publications/wzbrief-bildung. Zugegriffen am 25.10.2018.

Leemann, R. J., C. Imdorf, J. J. W. Powell, und M. Sertl, Hrsg. 2016a. Die Organisation von Bildung. Soziologische Analysen zu Schule, Berufsbildung, Hochschule und Weiterbildung. Weinheim/Basel: Beltz Juventa Verlag.

Leemann, R. J., C. Imdorf, J. J. W. Powell, und M. Sertl, Hrsg. 2016b. Die Organisation von Bildung aus soziologischer Perspektive. In Die Organisation von Bildung. Soziologische Analysen zu Schule, Berufsbildung, Hochschule und Weiterbildung, 9-11. Weinheim/Basel: Beltz Juventa Verlag.

Meyer, J. W., und B. Rowan. 2002. Institutionalized organizations: Formal structure as myth and ceremony. In The new institutionalism in organizational analysis, Hrsg. Walter W. Powell und Paul J. DiMaggio, 41-62. Chicago: University of Chicago Press.

Meyer, J. W., F. O. Ramirez, R. Rubinson, und J. Boli-Bennett. 1977. The world educational revolution, 1950-1970. Sociology of Education 50(4): 242-258.

Powell, W. W., und P. DiMaggio, Hrsg. 1991. The new institutionalism in organizational analysis. Chicago: University of Chicago Press.

Scott, W. Richard. 1995. Institutions and organizations. London: Sage. 